\title{
One-Step Synthesis and Bioassay of $N$-Phosphoramidophosphonates
}

\author{
Mudumala Veera Narayana Reddy, ${ }^{a}$ Avula Balakrishna, ${ }^{a}$ Mungara Anil Kumar, ${ }^{a}$ \\ Gangireddygari Chandra SeKhar Reddy, ${ }^{a}$ Arigela Uma Ravi Sankar, ${ }^{a}$ \\ Cirandur Suresh Reddy, ${ }^{*}, a$ and Tirupati Murali KrishnA ${ }^{b}$ \\ ${ }^{a}$ Department of Chemistry, Sri Venkateswara University; Tirupati 517 502, India: and ${ }^{b}$ Department of Entomology, \\ Regional Agricultural Research Station; Tirupati 517 502, India. \\ Received September 2, 2009; accepted September 24, 2009; published online September 28, 2009
}

\begin{abstract}
A simple one-step synthesis was accomplished for the preparation of $N$-phosphoramidophosphonates by a direct reaction of phosphoramidate (1) with heterocyclic aldehydes $(2 \mathrm{a}-\mathrm{j})$ and dialkyl phosphites at $60-70^{\circ} \mathrm{C}$ in the presence of tetramethylguanidine. The tetramethylguanidine not only catalyses this reaction but also helps to form pure products in high yields in lesser time. They exhibited good insecticidal and antioxidant properties.
\end{abstract}

Key words phosphoramidate; tetramethylguanidine; phosphoramidophosphonate; insecticidal activity; antioxidant activity

The organophosphorus (OP) compounds are one of the largest groups of insecticides in use today and have largely replaced the organochlorine insecticides. The universal use of organophosphorus insecticides has brought about increased benefits in crop production by minimizing pest damage and thereby allowing farmers to adopt better agronomic practices and reap high yields. The OP insecticides have two distinct features. Thy are acutely toxic with a high level of activity inhibiting the vital enzyme cholinesterase, and are chemically unstable and non-persistent in the environment, unlike the chlorinated hydrocarbons. ${ }^{1,2)}$ Several OP fungicides, such as iprobenfos and edifenphos, are used for the control of fungal disease in plant. ${ }^{3)}$ They are effective through their activity against chitin synthetase directly through inhibition of the methylation path of phosphatidylcholine synthesis. ${ }^{4}$

In spite of their great practical importance, the detailed mechanism of antioxidant action of organophosphorus compounds and their relationships between chemical structure and antioxidant activity have been comprehensively elucidated only in recent times. ${ }^{5,6)}$ Depending on their structure, the nature of the polymer to be stabilized and the aging conditions, phosphites and phosphonates may act both as sec- ondary and primary antioxidants.

In the present investigation, insecticidal, radical scavenging capacity and antioxidative activity for the newly synthesized compounds are evaluated using two antioxidant methodologies.

\section{Results and Discussion}

Reaction of 6-amino- $6 \lambda^{5}$-dibenzo[ $\left.d, f\right][1,3,2]$ dioxaphosphepin6-oxide 1 with various heterocyclic aldehydes $\mathbf{2 a}-\mathbf{j}$ and dialkyl phosphites in dry toluene in the presence of tetramethylguanidine (TMG) acts as a catalyst at $60-70^{\circ} \mathrm{C}$ for $4 \mathrm{~h}$ afforded $3 \mathbf{a}-\mathbf{j}^{7)}$ in good yields. In the absence of TMG the reaction takes $10 \mathrm{~h}$ and product yields are low. The same reaction in the presence of TMG, is completed in $4 \mathrm{~h}$ with high yields $79.2-91.6 \%$ (Table 1). The progress of the reaction was monitored by thin layer chromatography (TLC). The chemical structures of $\mathbf{3 a}-\mathbf{j}$ were confirmed by elemental analyses, IR, ${ }^{1} \mathrm{H}-,{ }^{13} \mathrm{C}-,{ }^{31} \mathrm{P}-\mathrm{NMR}$ and mass spectra.

Compounds $\mathbf{3 a}-\mathbf{j}$ exhibited characteristic IR stretching frequencies in the regions $3410-3380,1280-1230,1215-$ 1190 and $740-780 \mathrm{~cm}^{-1}$ for $\mathrm{N}-\mathrm{H}, \mathrm{P}=\mathrm{O}$ (phosphonate), $\mathrm{P}=\mathrm{O}$ (phosphepine) and $\mathrm{P}-\mathrm{C}$ (aliphatic) respectively. ${ }^{8)}$ The aromatic hydrogens in $\mathbf{3 a}-\mathbf{j}$ showed a complex multiplet at

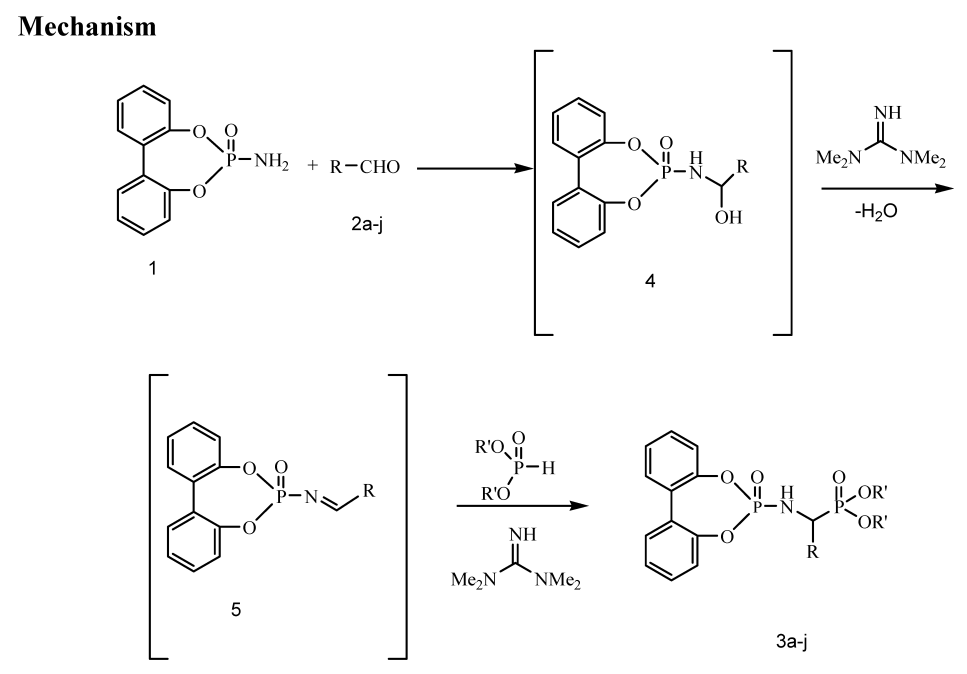

Forming the corresponding Schiff's base 5 

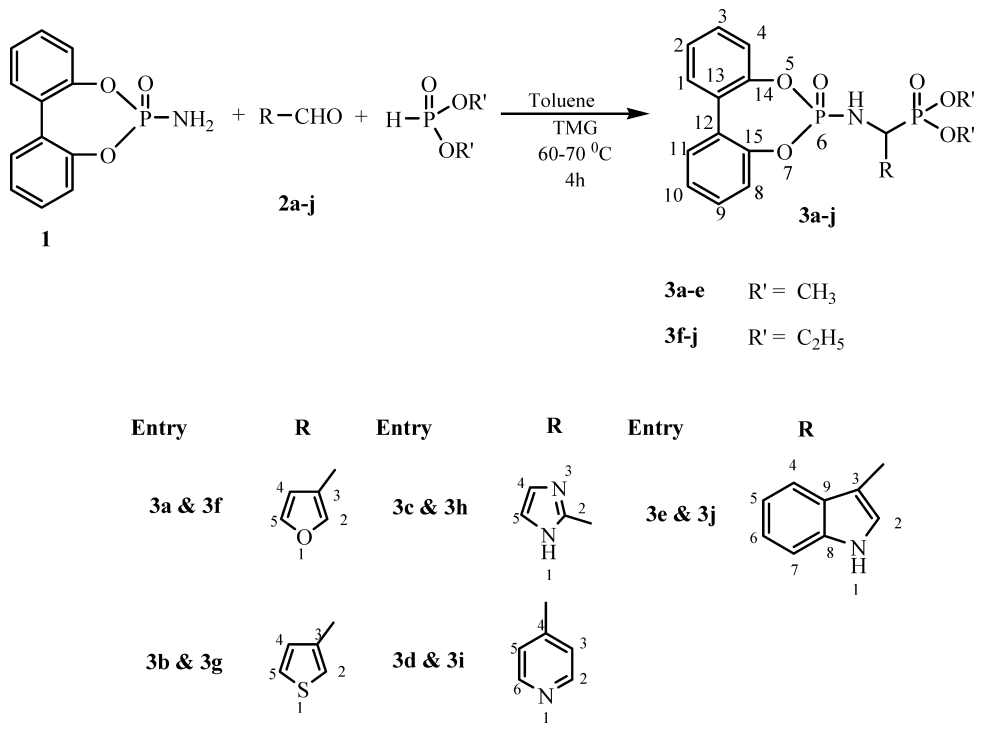

Chart 1

Table 1. TMG Promoted Synthesis of Phosphoramidate Phosphonates $\mathbf{3 a}-\mathbf{j}$

\begin{tabular}{|c|c|c|c|c|}
\hline Compounds & $\mathrm{R}$ & $\mathrm{R}^{\prime}$ & Yield $(\%)^{a)}$ & Yield $(\%)^{b)}$ \\
\hline $3 a$ & & $\mathrm{CH}_{3}$ & 79.2 & 62.2 \\
\hline $\mathbf{3 b}$ & & $\mathrm{CH}_{3}$ & 83.9 & 67.5 \\
\hline $3 c$ & & $\mathrm{CH}_{3}$ & 89.6 & 59.2 \\
\hline $3 d$ & & $\mathrm{CH}_{3}$ & 87.6 & 61.2 \\
\hline $3 e$ & & $\mathrm{CH}_{3}$ & 91.5 & 58.9 \\
\hline $3 f$ & & $\mathrm{CH}_{3} \mathrm{CH}_{2}$ & 79.7 & 62.5 \\
\hline $3 g$ & & $\mathrm{CH}_{3} \mathrm{CH}_{2}$ & 90.3 & 63.5 \\
\hline $3 h$ & & $\mathrm{CH}_{3} \mathrm{CH}_{2}$ & 91.6 & 59.8 \\
\hline $3 \mathbf{i}$ & & $\mathrm{CH}_{3} \mathrm{CH}_{2}$ & 87.1 & 61.2 \\
\hline $3 \mathbf{j}$ & & $\mathrm{CH}_{3} \mathrm{CH}_{2}$ & 90.9 & 62.9 \\
\hline
\end{tabular}

a) When the reactions were carried at $60-70^{\circ} \mathrm{C}$ in toluene for $1 \mathrm{~h}$ in the presence of TMG. b) When the reactions were carried at $115-120^{\circ} \mathrm{C}$ in toluene without catalyst for $10 \mathrm{~h}$.

$\delta 6.76-8.21$. The $\mathrm{P}-\mathrm{C}-\mathrm{H}$ hydrogen gave a doublet of doublet $^{9)}$ at $\delta 5.60-5.80\left(\mathrm{dd},{ }^{2} J=17.2 \mathrm{~Hz},{ }^{2} J=11.3 \mathrm{~Hz}\right)$ due to its coupling with phosphorus and the adjacent $\mathrm{NH}$ hydrogen.
The exocyclic $\mathrm{N}-\mathrm{H}$ hydrogen gave a singlet at $\delta 5.80-5.98$. The multiplet at $\delta 3.81-4.21$ and the triplet at $\delta 1.15-1.29$ $(\mathrm{t}, J=7.4 \mathrm{~Hz}), 1.08-1.16(\mathrm{t}, J=6.9 \mathrm{~Hz})$ were attributed to methylene and methyl hydrogens respectively. ${ }^{10)}$ The methoxy hydrogens of the dimethyl phosphate moiety resonated as two distinct doublets in the range of $\delta 3.65-3.89$ (d, $J=11.2 \mathrm{~Hz}) 3.35-3.55(\mathrm{~d}, J=10.2 \mathrm{~Hz})$ indicating their non-equivalence. ${ }^{11)}$

The carbon chemical shifts for $\mathrm{P}-\mathrm{CH}, \mathrm{P}-\mathrm{O}-\mathrm{CH}_{3}$ and $\mathrm{P}-\mathrm{O}-\mathrm{CH}_{2}-\mathrm{CH}_{3}$ were observed at $\delta 45.0,56.3,63.4$ and 16.4 respectively. ${ }^{9)}$ The two distinct ${ }^{31} \mathrm{P}$ chemical shifts appeared at $\delta 40.25$ and $\delta 2.86$ are assigned for exocyclic and endocyclo phosphorus. ${ }^{12)}$ Compounds $\mathbf{3 d}$, 3e, 3f, and $\mathbf{3 h}$ exhibited their respective molecular and characteristic daughter ion peaks in the mass spectra.

The merits of this procedure are: less reaction time, simple work-up and formation of relatively a pure product in high yields. TMG being a strong base $\left(\mathrm{p} K_{\mathrm{a}} 10.78\right)$ within ability to react with substrates by virtue of its structure, not only catalyses this reaction but also guides its course to avoid formation side products. Therefore, the use of TMG to perform the addition of dialkyl phosphates to unsaturated systems could offer significant advantages especially in terms of experimental simplicity, and easy work-up, high yield, lesser time, handling easy and could represent a convenient tool for the synthesis of a variety of phosphonate synthons.

Bioefficacy Activity Stomach Action: Results presented in Table 2 revealed that among the ten new compounds tested for their toxicity against Spodotera litura, 3b, 3c, $\mathbf{3 g}$ and $\mathbf{3 h}$ showed high larval mortality of 91.67, 91.67, 83.33 and $83.33 \%$ respectively after $72 \mathrm{~h}$ of the treatments, with reference to that of chloropyriphos. Compound $\mathbf{3 f}$ recorded $58.33 \%$ mortality and other compounds 3a, 3d, and $\mathbf{3 e}$ recorded $33.33 \%$ mortality. These results further revealed that four compounds $(\mathbf{3 b}, \mathbf{3 c}, \mathbf{3 g}, \mathbf{3 h})$ possess activity almost on par with chloropyriphos. This is observed that spraying the four compounds $(\mathbf{3 b}, \mathbf{3} \mathbf{c}, \mathbf{3 g}, \mathbf{3 h})$, on the foliage of crop surface gives complete control of $S$. litura on the third day after spraying. However, $S$. litura, feeding was reduced at the end of second day after spraying. Phytotoxicity symptoms 
Table 2. Percent Mortality of S. litura Larvae Due to Toxicity of Compounds $3 \mathbf{a}-\mathbf{j}$ by Stomach Action and Contact Action

\begin{tabular}{|c|c|c|c|c|c|}
\hline \multicolumn{3}{|c|}{ Stomach action } & \multicolumn{3}{|c|}{ Contact action } \\
\hline $\begin{array}{l}\text { Test Cc } \\
\text { compounds }\end{array}$ & $\begin{array}{l}\text { Concentration } \\
\quad(\mathrm{ppm})\end{array}$ & $\begin{array}{c}\text { Mean } \\
\text { mortality } \\
(\%)\end{array}$ & $\begin{array}{l}\text { Test Co } \\
\text { compounds }\end{array}$ & $\begin{array}{l}\text { Concentration } \\
\text { (ppm) }\end{array}$ & $\begin{array}{c}\text { Mean } \\
\text { mortality } \\
(\%)\end{array}$ \\
\hline $3 a$ & 2000 & $\begin{array}{c}33.33 \\
(35.00)\end{array}$ & $3 \mathbf{a}$ & 2000 & $\begin{array}{c}8.33 \\
(10.00)\end{array}$ \\
\hline $3 b$ & 2000 & $\begin{array}{c}91.67 \\
(75.00)\end{array}$ & $3 \mathbf{b}$ & 2000 & $\begin{array}{l}16.67 \\
(20.00)\end{array}$ \\
\hline $3 c$ & 2000 & $\begin{array}{c}83.33 \\
(75.00)\end{array}$ & $3 c$ & 2000 & $\begin{array}{l}16.67 \\
(25.00)\end{array}$ \\
\hline $3 d$ & 2000 & $\begin{array}{c}33.33 \\
(35.00)\end{array}$ & $3 d$ & 2000 & $\begin{array}{l}25.00 \\
(20.00)\end{array}$ \\
\hline $3 e$ & 2000 & $\begin{array}{c}33.33 \\
(35.00)\end{array}$ & $3 e$ & 2000 & $\begin{array}{l}25.00 \\
(20.00)\end{array}$ \\
\hline $3 f$ & 2000 & $\begin{array}{c}58.57 \\
(50.00)\end{array}$ & $3 f$ & 2000 & $\begin{array}{l}16.00 \\
(20.00)\end{array}$ \\
\hline $3 g$ & 2000 & $\begin{array}{l}91.67 \\
(80.00)\end{array}$ & $3 g$ & 2000 & $\begin{array}{l}33.33 \\
(35.00)\end{array}$ \\
\hline $3 \mathrm{~h}$ & 2000 & $\begin{array}{l}83.33 \\
(75.00)\end{array}$ & $3 \mathrm{~h}$ & 2000 & $\begin{array}{c}16.00 \\
(20.00)\end{array}$ \\
\hline $3 \mathbf{i}$ & 2000 & $\begin{array}{c}25.00 \\
(30.00)\end{array}$ & $3 \mathbf{i}$ & 2000 & $\begin{array}{l}20.33 \\
(25.33)\end{array}$ \\
\hline $3 \mathbf{j}$ & 2000 & $\begin{array}{l}25.00 \\
(30.00)\end{array}$ & $3 \mathbf{j}$ & 2000 & $\begin{array}{c}8.33 \\
(10.00)\end{array}$ \\
\hline Chloropyripho & Ios 2000 & $\begin{array}{l}100.00 \\
(90.00)\end{array}$ & Chloropyriphos & os 2000 & $\begin{array}{c}91.67 \\
(80.00)\end{array}$ \\
\hline $\begin{array}{c}\text { Control } \\
\text { (untreated) }\end{array}$ & & $\begin{array}{c}0.00 \\
(0.00)\end{array}$ & $\begin{array}{c}\text { Control } \\
\text { (untreated) }\end{array}$ & & $\begin{array}{c}0.00 \\
(0.00)\end{array}$ \\
\hline $\mathrm{CD}$ at $5 \%$ & & 37.76 & $\mathrm{CD}$ at $5 \%$ & & 31.88 \\
\hline S.E.M. \pm & & 12.87 & S.E.M. \pm & & 10.87 \\
\hline
\end{tabular}

were not observed with any other test compounds.

Contact action: Results presented in Table 2 revealed that the compound $\mathbf{3 g}$ recorded $33.33 \%$ larvae mortality followed by $3 \mathbf{d}$ and $3 \mathrm{e}$ which recorded $25 \%$ mortality at $72 \mathrm{~h}$ after spraying. Larvae mortality rate was very low in other compounds when compared with that of chloropyriphos with $91.67 \%$ mortality. Larvae feeding were normal but slow from third day after spraying.

Antioxidant Testing: The compounds $\mathbf{3 a}-\mathbf{j}$ were tested for antioxidant property by nitric oxide ${ }^{13,14)}$ and 1,1-diphenyl-2picrylhydrazyl (DPPH) ${ }^{15)}$ methods. The compounds $\mathbf{3 b}, \mathbf{3 c}$, $\mathbf{3 g}$ and $\mathbf{3 h}$ exhibited high antioxidant property in both nitric oxide and DPPH methods at $100 \mu \mathrm{m}$ concentrations (Table $3)$.

\section{Conclusion}

An elegant one-pot synthesis of pohsphoramidophosphonates $(\mathbf{3} \mathbf{a}-\mathbf{j})$ by one-step reaction of phospharamidate (1), heterocyclic aldehydes $(\mathbf{2} \mathbf{a}-\mathbf{j})$ and dialkyl phosphites using TMG as a catalyst is accomplished and good insecticidal and antioxidant properties.

\section{Experimental}

General Procedure The melting points were determined in open capillary tubes on a Mel-Temp apparatus and were uncorrected. The IR spectra $\left(v_{\max }\right.$ in $\mathrm{cm}^{-1}$ ) were recorded as $\mathrm{KBr}$ pellets on Perkin Elmer 1000 unit. The ${ }^{1} \mathrm{H}-,{ }^{13} \mathrm{C}$ - and ${ }^{31} \mathrm{P}-\mathrm{NMR}$ spectra were recorded on a Varian AMX $400 \mathrm{MHz}$ NMR spectrometers operating at $400 \mathrm{MHz}$ for ${ }^{1} \mathrm{H}-, 100.57 \mathrm{MHz}$ for ${ }^{13} \mathrm{C}$ - and $161.7 \mathrm{MHz}$ for ${ }^{31} \mathrm{P}$. All the compounds were dissolved in $\mathrm{CDCl}_{3}$ or DMSO$d_{6}$ and chemical shifts were referenced to tetramethyl silane (TMS) $\left({ }^{1} \mathrm{H},{ }^{13} \mathrm{C}\right)$ and $85 \% \mathrm{H}_{3} \mathrm{PO}_{4}\left({ }^{31} \mathrm{P}\right)$. Micro analyses data were obtained from Central Drug Research Institute, Lucknow, India.
Table 3. Nitric Oxide/DPPH Radical Scavenging Activity of $\mathbf{3 a}-\mathbf{j}$

\begin{tabular}{ccccc}
\hline \hline \multicolumn{2}{c}{ \% Inhibition at $100 \mu \mathrm{M}$} & & \multicolumn{2}{c}{ \% Inhibition at $100 \mu \mathrm{M}$} \\
\cline { 1 - 2 } \cline { 4 - 5 } $\begin{array}{c}\text { Test } \\
\text { compounds }\end{array}$ & $\begin{array}{c}\text { Nitric oxide } \\
\text { radical }\end{array}$ & & $\begin{array}{c}\text { Test } \\
\text { compounds }\end{array}$ & $\begin{array}{c}\text { DPPH } \\
\text { radical }\end{array}$ \\
\hline 3a & 65.25 & & $\mathbf{3 a}$ & 64.33 \\
3b & 85.24 & & $\mathbf{3 b}$ & 89.25 \\
3c & 90.11 & & $\mathbf{3 c}$ & 90.28 \\
3d & 60.36 & & $\mathbf{3 d}$ & 63.55 \\
3e & 65.30 & & $\mathbf{3 e}$ & 68.89 \\
3f & 65.23 & & $\mathbf{3 f}$ & 73.25 \\
3g & 88.25 & & $\mathbf{3 g}$ & 84.25 \\
3h & 89.36 & & $\mathbf{3 h}$ & 87.32 \\
3i & 68.36 & & $\mathbf{3 i}$ & 65.26 \\
3j & 69.69 & & $\mathbf{3 j}$ & 68.25 \\
BHT & 58.62 & BHT & 67.53 \\
\hline
\end{tabular}

6-Amino-6 $\lambda^{5}$-dibenzo $[d, f][1,3,2]$ dioxaphosphepin-6-oxide (1) The intermediate, 6 -chloro- $6 \lambda^{5}$-dibenzo $[d, f][1,3,2]$ dioxaphosphepin-6-oxide ${ }^{16)}$ $(0.05 \mathrm{~mol})$ was made to react with $\mathrm{NaNH}_{2}(0.05 \mathrm{~mol})$ in $20 \mathrm{ml}$ of dry toluene at $0{ }^{\circ} \mathrm{C}$ over a period of $30 \mathrm{~min}$. Then it was stirred for $5 \mathrm{~h}$ at $30^{\circ} \mathrm{C} 6$-amino$6 \lambda^{5}$-dibenzo $[d, f][1,3,2]$ dioxaphosphepin-6-oxide (1) was formed as indicated by TLC. The solvent was removed from the reaction mixture under reduced pressure. The residue was purified by column chromatography on silica gel ( 80 - 120 mesh) using petroleum ether-ethylacetate $(8: 2)$ as eluent. It was recrystallized from 2-propanol to afford pure 1 in $89 \%$ yield. mp $166-168^{\circ} \mathrm{C}$.

6-Oxo-6 $\lambda^{5}$-dibenzo[ $\left.d, f\right][1,3,2]$ dioxaphosphepin-6-yldiethyl $\{3$-(furylamino)methyl\}phosphonate (3a) Furyl 3-carboxaldehyde (2a) (0.005 $\mathrm{mol})$ in anhydrous toluene $(20 \mathrm{ml})$ and stoichiometric amount of TMG was added to the stirred solution of phosphoramide (1) $(0.005 \mathrm{~mol})$ in anhydrous toluene $(15 \mathrm{ml})$ at room temperature. After stirring for $30 \mathrm{~min}$, dimethyl phosphite $(0.005 \mathrm{~mol})$ in anhydrous toluene $(20 \mathrm{ml})$ was added dropwise. Stirring was continued for another $0.5 \mathrm{~h}$ at room temperature and then at $60-70^{\circ} \mathrm{C}$ for $3 \mathrm{~h}$. The progress of the reaction was monitored by TLC analysis. After completion of the reaction the solvent was removed under reduced pressure. The residue was purified by column chromatography on silica gel ( $80-120$ mesh) using petroleum-ethyl acetate $(8: 3)$ as eluent. It was recrystallized from 2-propanol to afford pure $\mathbf{3 a}$ in $79.2 \%$ yield. mp $189-191{ }^{\circ} \mathbf{C}$. Other compounds $(\mathbf{3} \mathbf{b}-\mathbf{j})$ were prepared by using this above procedure.

6-Oxo-6 $\lambda^{5}$-dibenzo[ $\left.d, f\right][1,3,2]$ dioxaphosphepine-6-yl-dimethyl-3-furyl Aminomethylphosphonate (3a): Colorless solid, $\mathrm{mp} 210-212^{\circ} \mathrm{C}$. IR $(\mathrm{KBr})$ $\mathrm{cm}^{-1}$ : $3420(\mathrm{NH}), 1270(\mathrm{P}=\mathrm{O}$, phosphonate), $1190(\mathrm{P}=\mathrm{O}$, phosphepine), $752\left(\mathrm{P}-\mathrm{C}_{\text {aliphatic }}\right) .{ }^{1} \mathrm{H}-\mathrm{NMR}\left(400 \mathrm{MHz}, \mathrm{DMSO}-d_{6}\right) \delta: 6.80-7.82(10 \mathrm{H}, \mathrm{m}$, $\mathrm{Ar}-\mathrm{H}), 5.72(\mathrm{~d}, J=8.9 \mathrm{~Hz}, \mathrm{Ar}-\mathrm{H}), 5.92(1 \mathrm{H}, \mathrm{s}, \mathrm{N}-\mathrm{H}), 5.72(1 \mathrm{H}, \mathrm{dd}$, $\left.{ }^{2} J_{\mathrm{P}-\mathrm{H}}=14.3 \mathrm{~Hz},{ }^{3} J_{\mathrm{P}-\mathrm{H}}=11.4 \mathrm{~Hz}\right), 3.68\left(3 \mathrm{H}, \mathrm{d},{ }^{3} J_{\mathrm{P}-\mathrm{H}}=10.2 \mathrm{~Hz}, \mathrm{P}-\mathrm{OCH}_{3}\right), 3.45$ $\left(3 \mathrm{H}, \mathrm{d},{ }^{3} J_{\mathrm{P}-\mathrm{H}}=9.2 \mathrm{~Hz}, \mathrm{P}-\mathrm{OCH}_{3}\right) .{ }^{31} \mathrm{P}-\mathrm{NMR}\left(161.7 \mathrm{MHz}, \mathrm{DMSO}-d_{6}\right) \delta: 28.2$ $(\mathrm{P}=\mathrm{O}$, phosphonate), $7.2 \quad(\mathrm{P}=\mathrm{O}$, phosphepine). Anal. Calcd for $\mathrm{C}_{19} \mathrm{H}_{19} \mathrm{NO}_{7} \mathrm{P}_{2} \mathrm{~S}: \mathrm{C}, 52.42 ; \mathrm{H}, 4.40 ; \mathrm{N}, 3.22$. Found: $\mathrm{C}, 52.38 ; \mathrm{H}, 4.36 ; \mathrm{N}$, $3.18 \%$.

6-Oxo-6 $\lambda^{5}$-dibenzo $[d, f][1,3,2]$ dioxaphosphepin-6-yl-dimethyl-3-thienyl Aminomethylphosphonate (3b): White solid, mp $180-182^{\circ} \mathrm{C}$; IR $(\mathrm{KBr})$ $\mathrm{cm}^{-1}$ : $3410(\mathrm{NH}), 1265(\mathrm{P}=\mathrm{O}$, phosphonate), $1210(\mathrm{P}=\mathrm{O}$, phosphepine); $756\left(\mathrm{P}-\mathrm{C}_{\text {aliphatic }}\right){ }^{1} \mathrm{H}-\mathrm{NMR}\left(400 \mathrm{MHz}, \mathrm{DMSO}-d_{6}\right) \delta: 6.71-7.83(11 \mathrm{H}, \mathrm{m}$, $\mathrm{Ar}-\mathrm{H}), 5.80(1 \mathrm{H}, \mathrm{s}, \mathrm{N}-\mathrm{H}), 5.60\left(1 \mathrm{H}, \mathrm{dd},{ }^{2} J=12.9 \mathrm{~Hz},{ }^{3} J_{\mathrm{P}-\mathrm{H}}=10.0 \mathrm{~Hz}\right), 3.65$ $\left.\left(3 \mathrm{H}, \mathrm{d},{ }^{3} \mathrm{~J}_{\mathrm{P}-\mathrm{H}}=11.4 \mathrm{~Hz}\right), \mathrm{P}-\mathrm{OCH}_{3}\right), 3.55\left(3 \mathrm{H}, \mathrm{d},{ }^{3} \mathrm{~J}_{\mathrm{P}-\mathrm{H}}=11.3 \mathrm{~Hz}, \mathrm{P}-\mathrm{OCH}_{3}\right)$. ${ }^{31} \mathrm{P}-\mathrm{NMR}\left(161.7 \mathrm{MHz}, \mathrm{DMSO}-d_{6}\right) \delta: 45.3(\mathrm{P}=\mathrm{O}$, phosphonate), $7.8(\mathrm{P}=\mathrm{O}$, phosphepine). Anal. Calcd. for $\mathrm{C}_{19} \mathrm{H}_{19} \mathrm{NO}_{6} \mathrm{P}_{2} \mathrm{~S}$ : C, 50.66; H, 4.24; N, 3.10. Found: C, 50.58; H, 4.18; N, $3.06 \%$.

6-Oxo-6 $\lambda^{5}$-dibenzo $[d, f][1,3,2]$ dioxaphosphepin-6-yl-dimethyl-1H-2-imidazol Aminomethylphosphonate (3c): White solid, $\mathrm{mp} 198-200^{\circ} \mathrm{C}$; IR $(\mathrm{KBr}) \mathrm{cm}^{-1}: 3390(\mathrm{NH}), 1260(\mathrm{P}=\mathrm{O}$, phosphonate), $1198(\mathrm{P}=\mathrm{O}$, phosphepine); 745 (P-C $\left.\mathrm{C}_{\text {aliphatic }}\right) .{ }^{1} \mathrm{H}-\mathrm{NMR}\left(400 \mathrm{MHz}, \mathrm{DMSO}-d_{6}\right) \delta: 9.89(1 \mathrm{H}$, s, imidazole N-H), 6.85-7.80 $(10 \mathrm{H}, \mathrm{m}, \mathrm{Ar}-\mathrm{H}), 5.90(1 \mathrm{H}, \mathrm{s}, \mathrm{N}-\mathrm{H}), 5.60(1 \mathrm{H}, \mathrm{dd}$, $\left.{ }^{2} J=14.2,{ }^{3} J_{\mathrm{P}-\mathrm{H}} 11.8 \mathrm{~Hz}, \mathrm{P}-\mathrm{CH}\right), 3.71\left(3 \mathrm{H}, \mathrm{d},{ }^{3} J_{\mathrm{P}-\mathrm{H}}=10.3 \mathrm{~Hz}, \mathrm{P}-\mathrm{OCH}_{3}\right), 3.62$ $\left(3 \mathrm{H}, \mathrm{d},{ }^{3} \mathrm{~J}_{\mathrm{P}-\mathrm{H}}=9.2 \mathrm{~Hz}, \mathrm{P}-\mathrm{OCH}_{3}\right) .{ }^{31} \mathrm{P}-\mathrm{NMR}\left(161.1 \mathrm{MHz}, \mathrm{DMSO}-d_{6}\right) \delta: 21.2$ ( $\mathrm{P}=\mathrm{O}$, Phosphonates), $3.2(\mathrm{P}=\mathrm{O}$, phosphepine). Anal. Calcd for $\mathrm{C}_{18} \mathrm{H}_{19} \mathrm{~N}_{3} \mathrm{O}_{6} \mathrm{P}_{2}$ : C, 49.67; H, 4.40; N, 9.65. Found: C, 49.62; H, 4.35; N, $9.60 \%$. 
6-Oxo-6 $\lambda^{5}$-dibenzo[ $\left.d_{2} f\right][1,3,2]$ dioxaphosphepin-6-yl-dimethyl-4-pyridyl Aminomethylphosphonate (3d): Colorless solid, mp 190-192 ${ }^{\circ} \mathrm{C}$. IR (KBr) $\mathrm{cm}^{-1}$ : $3419(\mathrm{NH}), 1226(\mathrm{P}=\mathrm{O}$, phosphonate), $1150(\mathrm{P}=\mathrm{O}$, phosphepine); $751\left(\mathrm{P}-\mathrm{C}_{\text {aliphatic }}\right) .{ }^{1} \mathrm{H}-\mathrm{NMR}\left(400 \mathrm{MHz}, \mathrm{DMSO}-d_{6}\right) \delta: 6.82-7.82(12 \mathrm{H}, \mathrm{m}$, Ar-H), $5.92(1 \mathrm{H}, \mathrm{s}, \mathrm{N}-\mathrm{H}), 5.62\left(1 \mathrm{H}, \mathrm{dd},{ }^{2} J_{\mathrm{P}-\mathrm{H}}=12.2 \mathrm{~Hz},{ }^{3} J_{\mathrm{P}-\mathrm{H}}=10.3 \mathrm{~Hz}\right.$, $\mathrm{P}-\mathrm{CH}), 3.65\left(3 \mathrm{H}, \mathrm{d},{ }^{3} J_{\mathrm{P}-\mathrm{H}}=12.8 \mathrm{~Hz}\right), 3.55\left(3 \mathrm{H}, \mathrm{d},{ }^{3} J_{\mathrm{P}-\mathrm{H}}=10.2 \mathrm{~Hz}, \mathrm{P}-\mathrm{OCH}_{3}\right)$. ${ }^{13} \mathrm{C}-\mathrm{NMR}\left(100 \mathrm{MHz}, \mathrm{CDCl}_{3}\right) \delta: 129.2(\mathrm{C}-1, \mathrm{C}-11), 122.6(\mathrm{C}-2, \mathrm{C}-10), 127.5$ (C-3, C-9), 118.3 (C-4, C-8), 126.1 (C-12, C-13), 149.9 (C-14, C-15), 149.5 $\left(\mathrm{C}^{\prime}-2, \mathrm{C}^{\prime}-6\right), 125.4\left(\mathrm{C}^{\prime}-3, \mathrm{C}^{\prime}-5\right), 138.8\left(\mathrm{C}^{\prime}-4\right), 51.5\left(\mathrm{~d},{ }^{2} J=7.3 \mathrm{~Hz}\right.$, $\left.\mathrm{P}-\mathrm{O}-\mathrm{CH}_{3}\right), 45.3(\mathrm{P}-\mathrm{CH}) .{ }^{31} \mathrm{P}-\mathrm{NMR}\left(161.7 \mathrm{MHz}, \mathrm{DMSO}-d_{6}\right) \delta: 28.6(\mathrm{P}=\mathrm{O}$, phosphonate), $2.6(\mathrm{P}=\mathrm{O}$, phosphepine). LC-MS; $m / z(\%) 447(60)(\mathrm{M}+1)$. Elemental Anal. Calcd for $\mathrm{C}_{20} \mathrm{H}_{20} \mathrm{~N}_{2} \mathrm{O}_{6} \mathrm{P}_{2}: \mathrm{C}, 53.82 ; \mathrm{H}, 4.52 ; 6.28$. Found: $\mathrm{C}$, $53.77 ; \mathrm{H}, 4.48 ; \mathrm{N}, 6.24 \%$.

6-Oxo-6 $\lambda^{5}$-dibenzo[ $\left.d, f\right][1,3,2]$ dioxaphosphepione-6-yl-dimethyl-3-indole Aminomethylphosphonate (3e): Pale yellow crystals, mp $190-192{ }^{\circ} \mathrm{C}$. IR $(\mathrm{KBr}) \mathrm{cm}^{-1}: 3435(\mathrm{NH}), 1267(\mathrm{P}=\mathrm{O}$, phosphonate), $1228(\mathrm{P}=\mathrm{O}$, phosphepine), $764\left(\mathrm{P}-\mathrm{C}_{\text {aliphatic }}\right) .{ }^{1} \mathrm{H}-\mathrm{NMR}\left(400 \mathrm{MHz}, \mathrm{DMSO}-d_{6}\right) \delta: 9.86(1 \mathrm{H}, \mathrm{s}$ Indole $\mathrm{N}-\mathrm{H}) \quad 6.81-8.15(13 \mathrm{H}, \mathrm{m}, \mathrm{Ar}-\mathrm{H}), 5.95(1 \mathrm{H}, \mathrm{s}, \mathrm{N}-\mathrm{H}), 5.69(1 \mathrm{H}, \mathrm{dd}$, $\left.{ }^{2} J=15.2,{ }^{3} J_{\mathrm{P}-\mathrm{H}}=11.6 \mathrm{~Hz}, \mathrm{P}-\mathrm{CH}\right), 3.65\left(3 \mathrm{H}, \mathrm{d},{ }^{3} J_{\mathrm{P}-\mathrm{CH}}=12.2 \mathrm{~Hz}, \mathrm{P}-\mathrm{OCH}_{3}\right)$, $3.40\left(3 \mathrm{H}, \mathrm{d},{ }^{3} J_{\mathrm{P}-\mathrm{CH}}=12.2 \mathrm{~Hz}, \mathrm{P}-\mathrm{OCH}_{3}\right) \cdot{ }^{13} \mathrm{C}-\mathrm{NMR}\left(100 \mathrm{MHz}, \mathrm{DMSO}-d_{6}\right) \delta$ : 130.3 (C-1, C-11), 122.4 (C-2, C-10), 129.9 (C-3, C-9), 119.9 (C-4, C-8), 126.4 (C-12, C-13), 150.3 (C-14, C-15), 121.7 (C'-2), 121.4 (C'-3) 123.6 $\left(C^{\prime}-4\right), 124.7\left(C^{\prime}-5\right), 123.6\left(C^{\prime}-6\right), 128.7\left(C^{\prime}-7\right), 139.9\left(C^{\prime}-8\right), 129.2\left(C^{\prime}-9\right)$, $53.2\left(\mathrm{~d},{ }^{2} J_{\mathrm{P}-\mathrm{C}} 6.5, \mathrm{P}-\mathrm{OCH}_{3}\right), 40.1(\mathrm{P}-\mathrm{CH}) .{ }^{31} \mathrm{P}-\mathrm{NMR}(161.7 \mathrm{MHz}$, DMSO$\left.d_{6}\right) \delta: 45.6(\mathrm{P}=0$, phosphonate $), 1.42(\mathrm{P}=\mathrm{O}$, phosphepine $)$. LC-MS; $m / z$ (\%), $486(30)(\mathrm{M}+2)$. Anal. Calcd for $\mathrm{C}_{23} \mathrm{H}_{22} \mathrm{~N}_{2} \mathrm{O}_{6} \mathrm{P}_{2}: \mathrm{C}, 57.03 ; \mathrm{H}, 4.58 ; \mathrm{N}$, 5.78. Found: C, 57.00; H, 4.54; N, 5.74\%.

6-Oxo-6 $\lambda^{5}$-dibenzo $[d, f][1,3,2]$ dioxaphosphepin-6-yl-diethyl-3-furyl Aminomethylphosphonate (3f): Colorless solid, mp 189-191 ${ }^{\circ} \mathrm{C}$. IR (KBr) $\mathrm{cm}^{-1}$ : $3410(\mathrm{NH}), 1259(\mathrm{P}=\mathrm{O}$, phosphonate), $1210(\mathrm{P}=\mathrm{O}$, phosphepine), $760\left(\mathrm{P}-\mathrm{C}_{\text {aliphatic }}\right) .{ }^{1} \mathrm{H}-\mathrm{NMR}\left(400 \mathrm{MHz}, \mathrm{DMSO}-d_{6}\right) \delta: 6.81-7.80(10 \mathrm{H}, \mathrm{m}$, $\mathrm{Ar}-\mathrm{H}), 5.98(1 \mathrm{H}, \mathrm{s}, \mathrm{NH}), 5.80(1 \mathrm{H}, \mathrm{d}, J=9.8 \mathrm{~Hz}, \mathrm{Ar}-\mathrm{H}), 5.60(1 \mathrm{H}, \mathrm{dd}$, $\left.{ }^{2} J_{\mathrm{P}-\mathrm{H}}=17.1 \mathrm{~Hz},{ }^{3} J_{\mathrm{P}-\mathrm{H}}=11.2 \mathrm{~Hz}, \mathrm{P}-\mathrm{CH}\right), 3.82-4.21\left(4 \mathrm{H}, \mathrm{m}, \mathrm{P}-\mathrm{OCH}_{2}-\mathrm{CH}_{3}\right)$, $1.15\left(3 \mathrm{H}, \quad \mathrm{t}, \quad{ }^{3} J=7.3 \mathrm{~Hz}, \quad \mathrm{P}-\mathrm{OCH}_{2}-\mathrm{CH}_{3}\right), \quad 1.10 \quad\left(3 \mathrm{H}, \quad \mathrm{t}, \quad{ }^{3} J=6.5 \mathrm{~Hz}\right.$, $\left.\mathrm{P}-\mathrm{OCH}_{2}-\mathrm{CH}_{3}\right) \cdot{ }^{13} \mathrm{C}-\mathrm{NMR}\left(100 \mathrm{MHz}\right.$, DMSO- $\left.d_{6}\right) \delta: 128.1$ (C-1, C-11), 120.1 (C-2, C-10), 128.1 (C-3, C-9), 118.1 (C-4, C-8), 128.2 (C-12, C-13), 151.2 (C-14, C-15), $139.4\left(\mathrm{C}^{\prime}-2\right), 120.8\left(\mathrm{C}^{\prime}-3\right), 109.2\left(\mathrm{C}^{\prime}-4\right), 146.8\left(\mathrm{C}^{\prime}-5\right)$, $64.5\left(\mathrm{~d},{ }^{2} J_{\mathrm{P}-\mathrm{C}}=7.6 \mathrm{~Hz}, \mathrm{P}-\mathrm{OCH}_{2} \mathrm{CH}_{3}\right), 46.1(\mathrm{P}-\mathrm{C}) 17.5\left(\mathrm{~d},{ }^{3} J_{\mathrm{P}-\mathrm{C}}=5.8 \mathrm{~Hz}\right.$, $\left.\mathrm{P}-\mathrm{OCH}_{2}-\mathrm{CH}_{3}\right) .{ }^{31} \mathrm{P}-\mathrm{NMR}\left(161.7 \mathrm{MHz}\right.$, DMSO- $\left.d_{6}\right) \delta: 28.2(\mathrm{P}=\mathrm{O}$, phosphonate), $3.80(\mathrm{P}=\mathrm{O}$, phosphepine). LC-MS; $m / z(\%): 486$ (100) $(\mathrm{M}+\mathrm{Na})$. Anal. Calcd for $\mathrm{C}_{21} \mathrm{H}_{23} \mathrm{NO}_{7} \mathrm{P}_{2}:$ C, 54.47; H, 5.00; N, 3.02 Found: C, 54.34; $\mathrm{H}, 4.96, \mathrm{~N}, 2.98 \%$.

6-Oxo-6 $\lambda^{5}$-dibenzo $[d, f][1,3,2]$ dioxaphosphepin-6-yl-diethyl-3-thineyl Aminomethylphosphonate (3g): Colorless solid, mp 191-193 ${ }^{\circ} \mathrm{C}$. IR (KBr) $\mathrm{cm}^{-1}$ : $3410(\mathrm{NH}), 1260(\mathrm{P}=\mathrm{O}$, phosphonate), $1190(\mathrm{P}=\mathrm{O}$, phosphepine $)$, $755\left(\mathrm{P}-\mathrm{C}_{\text {aliphatic }}\right) .{ }^{1} \mathrm{H}-\mathrm{NMR}\left(400 \mathrm{MHz}, \mathrm{DMSO}-d_{6}\right) \delta: 6.82-7.70(11 \mathrm{H}, \mathrm{m}$, $\mathrm{Ar}-\mathrm{H}), 5.93(1 \mathrm{H}, \mathrm{s}, \mathrm{NH}), 5.60\left(1 \mathrm{H}, \mathrm{d},{ }^{2} J_{\mathrm{P}-\mathrm{H}}=16.8 \mathrm{~Hz},{ }^{3} J_{\mathrm{P}-\mathrm{H}}=11.2 \mathrm{~Hz}\right.$, $\mathrm{P}-\mathrm{CH}), 3.81-4.10\left(4 \mathrm{H}, \mathrm{m}, \mathrm{P}-\mathrm{OCH}_{2}-\mathrm{CH}_{3}\right), 1.18\left(3 \mathrm{H}, \mathrm{t},{ }^{3} \mathrm{~J}=7.8 \mathrm{~Hz}\right.$, $\left.\mathrm{P}-\mathrm{OCH}_{2}-\mathrm{CH}_{3}\right), \quad 1.10 \quad\left(3 \mathrm{H}, \quad \mathrm{t}, \quad{ }^{3} \mathrm{~J}=6.6 \mathrm{~Hz}, \quad \mathrm{P}-\mathrm{OCH}_{2}-\mathrm{CH}_{3}\right) . \quad{ }^{31} \mathrm{P}-\mathrm{NMR}$ $\left(161.7 \mathrm{MHz}\right.$, DMSO- $\left.d_{6}\right) \quad \delta: 29.2 \quad(\mathrm{P}=\mathrm{O}$, phosphonate $), 2.90 \quad(\mathrm{P}=\mathrm{O}$, phosphepine). Anal. Calcd. for $\mathrm{C}_{21} \mathrm{H}_{23} \mathrm{NO}_{6} \mathrm{P}_{2} \mathrm{~S}$ : C, 52.61; H, 4.84; N, 2.92. Found: C, $52.58 ; \mathrm{H}, 4.79, \mathrm{~N}, 2.88 \%$.

6-Oxo-6 $\lambda^{5}$-dibenzo[ $\left.d, f\right][1,3,2]$ dioxaphosphepin-6-yl-diethyl-1 $H$-2-imidazolyl Aminomethylphosphonate (3h): Colorless solid, mp $191-193{ }^{\circ} \mathrm{C}$. IR $(\mathrm{KBr}) \mathrm{cm}^{-1}: 3380(\mathrm{NH}), 1250(\mathrm{P}=\mathrm{O}$, phosphonate), $1195(\mathrm{P}=\mathrm{O}$ phosphepine), $750\left(\mathrm{P}-\mathrm{C}_{\text {aliphatic }}\right) .{ }^{1} \mathrm{H}-\mathrm{NMR}\left(400 \mathrm{MHz}, \mathrm{DMSO}-d_{6}\right) \delta: 6.81-7.80(10 \mathrm{H}$, $\mathrm{m}, \operatorname{Ar}-\mathrm{H}), 5.90(1 \mathrm{H}, \mathrm{s}, \mathrm{NH}), 5.60\left(1 \mathrm{H}, \mathrm{dd},{ }^{2} J_{\mathrm{P}-\mathrm{H}}=17.5 \mathrm{~Hz},{ }^{3} J_{\mathrm{P}-\mathrm{H}}=10.8 \mathrm{~Hz}\right.$, $\mathrm{P}-\mathrm{CH}), \quad 3.79-4.10\left(4 \mathrm{H}, \mathrm{m}, \mathrm{P}-\mathrm{OCH}_{2} \mathrm{CH}_{3}\right), 1.18 \quad\left(3 \mathrm{H}, \mathrm{t},{ }^{3} \mathrm{~J}=7.4 \mathrm{~Hz}\right.$, $\left.\mathrm{P}-\mathrm{OCH}_{2}-\mathrm{CH}_{3}\right), \quad 1.08 \quad\left(3 \mathrm{H}, \quad \mathrm{t}, \quad{ }^{3} \mathrm{~J}=6.6 \mathrm{~Hz}, \quad \mathrm{P}-\mathrm{OCH}_{2}-\mathrm{CH}_{3}\right) .{ }^{13} \mathrm{C}-\mathrm{NMR}$ $\left(100 \mathrm{MHz}, \mathrm{DMSO}-d_{6}\right) \delta: 129.2(\mathrm{C}-1, \mathrm{C}-11), 120.8(\mathrm{C}-2, \mathrm{C}-10), 129.8(\mathrm{C}-3$, C-9), 117.8 (C-4, C-8), 127.8 (C-12, C-13), 149.8 (C-14, C-15), 148.9 (C'2), $125.3\left(\mathrm{C}^{\prime}-4\right) 126.5\left(\mathrm{C}^{\prime}-5\right), 63.5\left(\mathrm{~d},{ }^{2} J_{\mathrm{POC}}=7.3 \mathrm{~Hz}, \mathrm{P}-\mathrm{OCH}_{2}-\mathrm{CH}_{3}\right), 45.1$ $(\mathrm{P}-\mathrm{C}) ; 15.3\left(\mathrm{~d},{ }^{3} J_{\mathrm{POCC}}=6.1 \mathrm{~Hz}, \mathrm{P}-\mathrm{OCH}_{2}-\mathrm{CH}_{3}\right) .{ }^{31} \mathrm{P}-\mathrm{NMR}(161.7 \mathrm{MHz}$, DMSO- $\left.d_{6}\right) \delta: 28.1(\mathrm{P}=\mathrm{O}$, phosphonate), $6.8(\mathrm{P}=\mathrm{O}$, phosphepine). LC-MS MS; $m / z(\%) 463(55)(\mathrm{M}+\mathrm{H})$. Anal. Calcd for $\mathrm{C}_{20} \mathrm{H}_{23} \mathrm{~N}_{3} \mathrm{O}_{6} \mathrm{P}_{2}: \mathrm{C}, 51.84 ; \mathrm{H}$, 5.00 ; N, 9.07. Found: C, 51.79; H, 4.95, N, 9.02\%.

6-Oxo-6 $\lambda^{5}$-dibenzo $[d, f][1,3,2]$ dioxaphosphepin-6-yl-diethyl-4-pyridyl Aminomethylphosphonate (3i): Colorless solid, mp $188-190^{\circ} \mathrm{C}$. IR $(\mathrm{KBr})$ $\mathrm{cm}^{-1}: 3420(\mathrm{NH}), 1240(\mathrm{P}=\mathrm{O}$, phosphonate), $1208(\mathrm{P}=\mathrm{O}$, phosphepine $)$, $759\left(\mathrm{P}-\mathrm{C}_{\text {aliphatic }}\right) .{ }^{1} \mathrm{H}-\mathrm{NMR}\left(400 \mathrm{MHz}, \mathrm{DMSO}-d_{6}\right) \delta: 6.82-8.12(12 \mathrm{H}, \mathrm{m}$, $\mathrm{Ar}-\mathrm{H}), 5.92(1 \mathrm{H}, \mathrm{s}, \mathrm{NH}), 5.63\left(1 \mathrm{H}, \mathrm{dd},{ }^{2} J_{\mathrm{P}-\mathrm{H}}=17.2 \mathrm{~Hz},{ }^{3} J_{\mathrm{P}-\mathrm{H}}=11.3 \mathrm{~Hz}\right.$, $\mathrm{P}-\mathrm{CH}), 3.72-3.98\left(4 \mathrm{H}, \mathrm{m}, \mathrm{P}-\mathrm{OCH}_{2}-\mathrm{CH}_{3}\right), 1.16\left(3 \mathrm{H}, \mathrm{t},{ }^{3} \mathrm{~J}=7.5 \mathrm{~Hz}\right.$, $\left.\mathrm{P}-\mathrm{OCH}_{2}-\mathrm{CH}_{3}\right), \quad 1.08 \quad\left(3 \mathrm{H}, \quad \mathrm{t}, \quad{ }^{3} \mathrm{~J}=6.4 \mathrm{~Hz}, \quad \mathrm{P}-\mathrm{OCH}_{2}-\mathrm{C}_{3}\right) . \quad{ }^{31} \mathrm{P}-\mathrm{NMR}$ (161.7 MHz, DMSO- $\left.d_{6}\right) \delta: 35.2(\mathrm{P}=\mathrm{O}$, phosphonate), $4.8(\mathrm{P}=\mathrm{O}$, phosphep- ine). Anal. Calcd. for $\mathrm{C}_{22} \mathrm{H}_{24} \mathrm{~N}_{2} \mathrm{O}_{6} \mathrm{P}_{2}: \mathrm{C}, 55.70 ; \mathrm{H}, 5.10 ; \mathrm{N}, 5.91$. Found: $\mathrm{C}$, $55.65 ; \mathrm{H}, 5.05 ; \mathrm{N}, 5.87 \%$.

6-Oxo-6 $\lambda^{5}$-dibenzo $[d, f][1,3,2]$ dioxaphosphepin-6-yl-diethyl-1 $H$-3-indolyl Aminomethylphosphonate (3j): Colorless solid, mp 198-200 ${ }^{\circ} \mathrm{C}$. IR $(\mathrm{KBr})$ $\mathrm{cm}^{-1}: 3410(\mathrm{NH}), 1280(\mathrm{P}=\mathrm{O}$, phosphonate), $1211(\mathrm{P}=\mathrm{O}$, phosphepine $)$; $746\left(\mathrm{P}-\mathrm{C}_{\text {aliphatic }}\right) .{ }^{1} \mathrm{H}-\mathrm{NMR}\left(400 \mathrm{MHz}, \mathrm{DMSO}-d_{6}\right) \delta: 6.80-8.10(13 \mathrm{H}, \mathrm{m}$, Ar-H), $5.91(1 \mathrm{H}, \mathrm{s}, \mathrm{NH}), 5.61\left(1 \mathrm{H}, \mathrm{dd},{ }^{2} J_{\mathrm{P}-\mathrm{H}}=17.2 \mathrm{~Hz},{ }^{3} J_{\mathrm{P}-\mathrm{H}}=11.2 \mathrm{~Hz}\right.$, $\mathrm{P}-\mathrm{CH}), 3.82-3.98\left(4 \mathrm{H}, \mathrm{m}, \mathrm{P}-\mathrm{OC}_{2}-\mathrm{CH}_{3}\right), 1.15\left(3 \mathrm{H}, \mathrm{t},{ }^{3} \mathrm{~J}=7.4 \mathrm{~Hz}\right.$, $\left.\mathrm{P}-\mathrm{OCH}_{2}-\mathrm{CH}_{3}\right), \quad 1.10 \quad\left(3 \mathrm{H}, \quad \mathrm{t}, \quad{ }^{3} J=6.6 \mathrm{~Hz}, \quad \mathrm{P}-\mathrm{OCH}_{2}-\mathrm{CH}_{3}\right) . \quad{ }^{31} \mathrm{P}-\mathrm{NMR}$ $\left(161.7 \mathrm{MHz}, \mathrm{DMSO}-d_{6}\right) \delta: 33.2(\mathrm{P}=\mathrm{O}$, phsophonate), $4.8(\mathrm{P}=\mathrm{O}$, phsophepine). Anal. Calcd for $\mathrm{C}_{25} \mathrm{H}_{26} \mathrm{~N}_{2} \mathrm{O}_{6} \mathrm{P}_{2}: \mathrm{C}, 58.60 ; \mathrm{H}, 5.11 ; \mathrm{N}, 5.47$. Found: $\mathrm{C}$, $58.56 ; \mathrm{H}, 5.08, \mathrm{~N}, 5.42 \%$.

Bioassay. Insecticidal Activity Compounds $\mathbf{3 a}-\mathbf{j}$ were tested for their bioefficacy ${ }^{17,18)}$ on tobacco caterpillar, Spodoptera litura. S. litura is a major pest on many crops like groundnut, cowpea, greengram, castor, and cotton, etc. Each compound was dissolved in mercapto ethanol for preparing the stock solutions and the compounds were tested at $2000 \mathrm{ppm}$ each. Chloropyriphos an organophosphorus insecticide is used as reference compound for the control of S. litura.

There were twelve treatments including a control which was maintained without insecticide application. Each treatment consisted of eight third instar larvae with three replications. Just before the insecticidal treatment the larvae were transferred carefully with a hair brush into a clean and dry Petri dish of $100 \times 15 \mathrm{~mm}$ at the rate of eight larvae per Petri dish covered with lids.

Two methods of testing were done to know the contact and stomach action of the compounds on S. litura. In one method castor leaves were cut into a disc size of $100 \times 15 \mathrm{~mm}$ so as to spread in the Petri dish. The compounds $\mathbf{3} \mathbf{a}-\mathbf{j}$ and chloropyriphos were sprayed on to the leaf disc before feeding it to the larvae in the Petri dish for the stomach action. In another method the compounds were sprayed directly on to the body of larvae kept in the Petri dish and later on transferred to a clean Petri dish with fresh castor leaf disc for determining the contact action. Potters' spraying tower was used to spray the insecticide in both the methods. Larvae mortality counts were taken at regular interval of $24 \mathrm{~h}$ after imposing the treatments. The larvae which were moribund and did not show any movement were considered as dead.

Antioxidant Testing The compounds $\mathbf{3 a}-\mathbf{j}$ are tested for antioxidant property by nitric oxide and DPPH methods.

Assay for Nitric Oxide (NO) Scavenging Activity Sodium nitroprusside $(5 \mu \mathrm{M})$ in phosphate buffer $\mathrm{pH} 7.4$ was incubated with $100 \mu \mathrm{M}$ concentration of test compounds dissolved in a suitable solvent (dioxane/methanol) and tubes were incubated at $25^{\circ} \mathrm{C}$ for $120 \mathrm{~min}$. Control experiment was conducted with equal amount of solvent in an identical manner. At intervals, $0.5 \mathrm{ml}$ of incubation solution was taken and diluted with $0.5 \mathrm{ml}$ of griess reagent $(1 \%$ sulfanilamide, $0.1 \% \mathrm{~N}$-naphthylethylenediamine dihydrochloride and $2 \% O$-phosphoric acid dissolved in distilled water). The absorbance of the chordophone formed during diazotization of nitrite with sulfanilamide and subsequent $N$-naphthylethylenediamine dihydrochloride was read at $546 \mathrm{~nm}$. The experiment was repeated in triplicate.

Reduction of 1,1-Diphenyl-2-picrylhydrazyl (DPPH) Free Radical (DPPH Method) The nitrogen centered stable free radical 1,1-diphenyl-2picrylhydrazyl (DPPH) has often been used to characterize antioxidants. It is reversibly reduced and the odd electron in the DPPH free radical gives a strong absorption maximum at $\lambda 517 \mathrm{~nm}$, which is purple in color. This property makes it suitable for spectrophotometric studies. A radical scavenging antioxidant reacts with DPPH stable free radical and converts into 1,1diphenyl-2-picrylhydrazine. The resulting decolorization is stoichiometric, with respect to the number of electrons captured. The change in the absorbance produced in this reaction has been used to measure antioxidant properties.

The solutions of test compounds $(100 \mu \mathrm{M})$ were added to DPPH $(100 \mu \mathrm{M})$ in dioxane/ethanol. The tubes were kept at an ambient temperature for $20 \mathrm{~min}$ and the absorbance was measured at $\lambda 517 \mathrm{~nm}$. The difference between the test and the control experiments was taken and expressed as the per cent scavenging of the DPPH radical.

Acknowledgements The authors thank Prof. C.D. Reddy, Dept. of Chemistry, S.V. University, Tirupati for helpful discussions and for UGC (33-299) New Delhi for providing financial assistance. The authors also express their thanks to Prof. Ch. Appa Rao and S. Swapna, Dept. of Biochemistry, S.V. University, Tirupati for conducting antioxidant activity.

\section{References}

1) Srivastava R. P., Saxena R. C., "Test Book of Insect Toxicology,” Hi- 
machal Publications, Udaipur, India, 1989, p. 165.

2) Perry A. S., Yamamoto I., Ishaye I., Perry R., "Insecticides in Agriculture and Environment: Retrospect and Prospects," Narosa Publishing House, New Delhi, 1998, p. 261.

3) Nene Y. L., Thapliya P. N., "Fungicides in Plant Disease Control," 2nd ed., Oxford \& IBH Publishing Co., New Delhi, 1979, p. 287.

4) "Antifungal Agents Discovery and Mode of Action," ed. by Dixon G. K., Copping L. G., Hollomon D. W., BIOS Scientific, Oxford and London, 1989, p. 77.

5) Schwetlick K., "Mechanisms of Polymer of Degradation and Stabillisation," ed. by Elsevier Applied Science, London and New York, 1990, p. 23.

6) Schwetlick K., Pure Appl. Chem., 55, 1629-1636 (1983).

7) Zhanwei C., Jianfeng Z., Fei W., Yadan W., Zhiwei M., Ruyu C., Carbohydr. Res., 343, 2530-2534 (2008).

8) Silverstein R. M., Webster F. X., "Spectrometric Identification of Organic Compounds," 6th ed., Wiley, New York, 1998.

9) Kumar B. S., Sankar A. U. R., Reddy M. V., Krishna A. B., Reddy C. S., Bulgarian Chemical Communications, 40, 59-64 (2008).
10) Jie W., Wei S., Hong-Guang X., Xiaoyu S., Org. Biomol. Chem., 4, 1663-1666 (2006).

11) Narayana Reddy M. V., Sivakumar B., Balakrishna A., Reddy C. S., Nayak S. K., Reddy C. D., Arkivoc, 15, 246-254 (2007).

12) Quin L. D., Verkade J. G., "Phosphorus-31 NMR Spectral Properties in Compound Characterization and Structural Analysis," VCH, New York, 1994.

13) Shirwaiker A., Rajendran K., Dinesh Kumar C., Indian J. Expl. Biol., 42, 803-807 (2004).

14) Babu B. H., Shailesh B. S., Paddikala J., Fitotherapia, 72, 272-279 (2001).

15) Kato K., Terao S., Shimamoto N., Hirata M., J. Med. Chem., 31, 793798 (1988).

16) Haranath P., Sreedhar Kumar V., Naga Raju C., Suresh Reddy C., Reddy C. D., Synthetic Commun., 37, 1697-1708, (2007).

17) Busvine Jr., "A Critical Review on the Techniques for Testing Insecticides," 6th ed., CAB, Slough, U.K., 1971, p. 345.

18) Snedecor G. W., Cochran W. G., "Statistical Methods," Iowa State University Press, Iowa, 1968, p. 533. 